\title{
Learning styles among first year medical students: Are we addressing the diversity?
}

\author{
Mathangasinghe $\mathrm{Y}^{1}$, Dissanayake $\mathrm{MM}^{1}$ \\ ${ }^{\prime}$ Department of Anatomy, Faculty of Medicine, University of Colombo, Colombo, Sri Lanka
}

\section{Introduction}

Teaching Anatomy for the first-year medical students is challenging. Each individual has their own preferred methods in learning. Therefore, we intended to study learning styles among first year medical students.

\section{Methods}

A descriptive cross-sectional study was conducted among first year medical students of the Faculty of Medicine, University of Colombo. The study conformed to the guidelines of the declaration of Helsinki and was approved by the Ethics Review Committee, Faculty of Medicine.

Learning styles of the students were assessed using Visual Auditory Read/write and Kinesthetic (VARK) questionnaire, which is a validated questionnaire to assess the predominant learning preferences based on four sensory modalities.

\section{Results}

Of 100 respondents, $36 \%(n=36)$ were males and $64 \%(n=64)$ were females. Mean age was $21.8 \pm 1.1$ years. Majority $(94 \%, n=94)$ were Sri Lankans while six students $(6 \%)$ were Bhutanese. None of them used English as their first language. Majority were multimodal learners $(65 \%, \mathrm{n}=65)$ followed by aural $(15 \%$, $\mathrm{n}=15)$, kinesthetic $(11 \%, \mathrm{n}=11)$, $\mathrm{read} / \mathrm{write}(5 \%, \mathrm{n}=5)$ and visual $(4 \%$, $\mathrm{n}=4)$. Sub group analysis of learning styles based on the affinity of the particular style (e.g. mild aural, moderate aural and severe aural) was not performed due to small number of the population. A Pearson's Chi square test of independence was calculated to compare the frequency of learning styles in males and females. There was no significant interaction between the genders $(\chi 2(4)=1.29, p=.86)$.

\section{Discussion and Conclusions}

Learning styles among first year medical students were diverse. Unimodal learners were rare. Therefore, a multimodal teaching approach to stimulate multiple senses should be employed in teaching activities in Anatomy for the first-year students. 